\title{
Gewaltenteilung als Mittel zur Konzeptualisierung von Partizipation
}

\author{
Dominik Steiger
}

Spätestens seit den Protesten gegen das Bahnhofsprojekt Stuttgart 21 ist Partizipation der Bürger an der Staatsgewalt in aller Munde. ${ }^{1}$ Der Gesetzgeber hat den Forderungen der Bürger nach mehr Mitgestaltungsmöglichkeiten in den letzten Jahren nicht tatenlos zugeschaut, sondern hat auf diese Entwicklungen reagiert und bestehende Partizipationsmöglichkeiten entweder erweitert oder neue geschaffen. Dazu gehören etwa Ausweitungen von Formen direkter Demokratie auf Länderebene ${ }^{2}$ oder der neue $\S 25$ Abs. $3 \mathrm{VwVfG}$, der die frühe Bürgerbeteiligung aus dem Baugesetzbuch in das Verwaltungsverfahrensgesetz überträgt. ${ }^{3}$

Die Diskussion um Partizipation konzentriert sich jeweils nur auf einen Zweig der Staatsgewalt. So wird einerseits untersucht, wie die partielle Ersetzung von Parlamentsgesetzgebung im Wege direkter Demokratie rechtlich ausgestaltet sein muss und inwiefern das Volk bei der Gesetzgebung zu beteiligen ist. ${ }^{4}$ Ebenso ist Gegenstand intensiver wissenschaftlicher De-

1 Aus der umfangreichen rechtswissenschaftlichen Literatur zu Stuttgart 21 siehe z.B. T. Groß, Stuttgart 21: Folgerungen für Demokratie und Verwaltungsverfahren, DÖV 64 (2011), 510; C. Franzius, Stuttgart 21: Eine Epochenwende?, GewArch 58 (2012), 225; F. Wittreck, Demokratische Legitimation von Großvorhaben, ZG 26 (2011), 209; K. F. Gärditz, Angemessene Öffentlichkeitsbeteiligung bei Infrastrukturplanungen als Herausforderung an das Verwaltungsrecht im demokratischen Rechtsstaat, GewArch 57 (2011), 273.

2 Siehe z.B. das Gesetz zur Änderung der Verfassung des Saarlandes zur Stärkung der Bürgerbeteiligung, 15. Mai 2013, Amtsbl. I, 178.

3 Siehe dazu $W$. Hertel/C.-D. Munding, ,Frühe Öffentlichkeitsbeteiligung“ bei Planung von Großvorhaben, NJW 65 (2012), 2622; J. Ziekow, Frühe Öffentlichkeitsbeteiligung, NVwZ 32 (2013), 754.

4 Aus der umfangreichen Literatur siehe z.B. M. Möstl, Elemente direkter Demokratie als Entwicklungsperspektive, VVDStRL 72 (2013), 355; M. Schuler-Harms, Elemente direkter Demokratie als Entwicklungsperspektive, VVDStRL 72 (2013), 417; R. Lehner, Direkte Demokratie und Gruppenrechte - Probleme der Kollektivierung individueller Partizipation in plebiszitären Rechtsetzungsverfahren, in: Junge Wissenschaft im Öffentlichen Recht (Hrsg.), Kollektivität - Öffentliches Recht 
batten, wie hoch der Einfluß der Bürger auf Verwaltungsentscheidungen sein darf. ${ }^{5}$ Schließlich wird Partizipation auch in einem dritten Zusammenhang diskutiert, auch wenn man sich oft gar nicht im Klaren ist, überhaupt an der Partizipationsdebatte teilzunehmen: Dies betrifft die Rechtsprechung und hier v.a. die Auseinandersetzungen um die Ausweitung der Klagebefugnisse in der Verwaltungsgerichtsbarkeit. Mit dieser Beschränkung auf nur eine Gewalt kann es aber nicht gelingen, die der Partizipation zugrunde liegenden Mechanismen zu verstehen. Vielmehr ist eine holistische Herangehensweise notwendig, die alle drei Staatsgewalten umfasst. Nur so kann Partizipation kohärent gestaltet werden. Dies ist zwar bislang zumeist der Fall, aber eben nur zumeist.

Schon anhand dieser kurzen Einleitung wird deutlich, dass hier ein Partizipationsbegriff zugrunde gelegt wird, der sich auf alle drei Gewalten bezieht. In einem ersten Schritt wird dieser Begriff aus dem Grundgesetz entwickelt (A.). In einem zweiten Schritt wird anhand der verschiedenen Funktionen von Partizipation nachgewiesen, dass Partizipation sowohl auf dem Demokratieprinzip als auch auf dem Rechtsstaatsprinzip beruht (B.). Daran anschließend wird der Blick in einem dritten Schritt der Gewaltenteilung zugewendet. Das Gewaltenteilungsprinzip löst das zwischen Demokratie- und Rechtsstaatsprinzip bestehende Spannungsverhältnis auf, indem es die verschiedenen Staatsaufgaben bestimmten Organen zuordnet (C.). In einem vierten Schritt werden die Voraussetzungen herausgearbeitet, unter denen der Gewaltenteilungsgrundsatz auch hinsichtlich von Partizipation die Spannung beider Prinzipien auflösen kann (D.). Damit sollen die zwei entscheidenden Fragen der Partizipationsdebatte beantwortet werden: Erstens, wer ist an der Ausübung öffentlicher Gewalt zu beteiligen und zweitens, welche Rechtswirkung soll von der Beteiligung ausgehen. Nach einem Fazit (E.) wird abschließend in einem Ausblick schlag-

zwischen Gruppeninteressen und Gemeinwohl, 2012, 271; P. Neumann, Sachunmittelbare Demokratie: im Bundes- und Landesverfassungsrecht unter besonderer Berücksichtigung der neuen Länder, 2009; J. Rux, Direkte Demokratie in Deutschland: Rechtsgrundlagen und Rechtswirklichkeit der unmittelbaren Demokratie in der Bundesrepublik Deutschland und ihren Ländern, 2008; P. M. Huber, Volksgesetzgebung und Ewigkeitsgarantie, 2003.

5 Aus der umfangreichen Literatur siehe z.B. K. Waechter, Großvorhaben als Herausforderung für den demokratischen Rechtsstaat, VVDStRL 72 (2013), 499; T. Mann, Großvorhaben als Herausforderung für den demokratischen Rechtsstaat, VVDStRL 72 (2013), 544; siehe auch die Nachweise in Fn. 1 und 3. 
lichtartig untersucht, welche Folgen sich aus diesem Partizipationskonzept für Exekutive, Judikative und Legislative ergeben können (F.).

\section{A. Partizipation im Spannungsverhältnis von Demokratie- und Rechtsstaatsprinzip}

Was ist unter Partizipation zu verstehen? Partizipation ist kein gängiger Begriff des positiven Rechts. ${ }^{6}$ Im Verwaltungsrecht wird gelegentlich zumindest das deutsche Synonym, nämlich „Beteiligung“, verwendet. ${ }^{7}$ Will man die vielschichtigen Funktionen von Partizipation verstehen, so darf der Begriff aber nicht nur verwaltungsrechtlich verstanden werden. Vielmehr muss er vom Grundgesetz her gedacht werden. Partizipation existiert in vielerlei Formen ${ }^{8}$ und betrifft die Teilhabe an allen drei Staatsgewalten.

Das Grundgesetz regelt die Ausübung staatlicher Gewalt, betrifft also die staatliche Sphäre. Dementsprechend muss sich auch Partizipation unter dem Grundgesetz auf die staatliche Ebene beziehen. Damit wird deutlich, dass der Partizipationsbegriff sich durch eine enge Verknüpfung mit der Ausübung staatlicher Gewalt auszeichnet. Partizipation ist also nur solches Handeln, das rechtsförmiges Innehaben oder Teilhaben an der Ausübung von Staatsgewalt darstellt. Durch Partizipation wird entweder unmittelbar Staatsgewalt ausgeübt - durch Wahlen und Abstimmungen entscheidet das Volk selbst und unmittelbar. Oder der Staat wird durch Partizipation im Vorfeld der Ausübung von Staatsgewalt zu einer rechts- und verfahrensförmigen Reaktion, die selbst wiederum Ausübung von Staatsgewalt darstellt, gezwungen. Dies geschieht etwa durch Anhörungen, Einwendungen aber auch Klagen. Davon abzugrenzen sind Beteiligungsformen, die den Staat nicht zu einer rechtsförmigen Reaktion zwingen. Damit fallen etwa

6 Er wird ausschließlich in Landesgesetzen verwendet. Dazu gehören v.a. solche, die auf Integration von Migranten abzielen, wie etwa das Gesetz zur Regelung von Partizipation und Integration in Berlin, GVBl. Berlin, 28. Dezember 2010. Das Gesetz zur Weiterentwicklung der politischen Partizipation in den Gemeinden und zur Änderung kommunalverfassungsrechtlicher Vorschriften, GV NRW, 30. Dezember 2013 führt den Begriff der Partizipation nur im Titel.

7 Siehe u.a. $\$ 9$ NABEG, $\S 85$ WHG.

8 Für einen politischen Begriff der Partizipation s. M. Steinbrecher, Politische Partizipation in Deutschland, 2009, $27 \mathrm{ff}$., für einen sozialen Begriff der Partizipation $S$. Roßteutscher, Soziale Partizipation und Soziales Kapital“, in: V. Kaina/A. Römmele (Hrsg.), Politische Soziologie, 163 (163). 
soziale Beteiligungsformen aus dem Partizipationsbegriff heraus. Das Gleiche gilt für Demonstrationen. Zwar ist die Versammlungsfreiheit zwingend notwendig für das demokratische Zusammenleben, ${ }^{9}$ jedoch muss der Staat nicht auf Versammlungen reagieren. Runde Tische und ähnliches erfordern ebenso wenig eine staatliche Reaktion und sind daher ebenfalls keine Partizipation im Sinne des Grundgesetzes.

Von diesem Partizipationsbegriff, der aufgrund des mit ihm einhergehenden Zwangs - entweder wird Staatsgewalt unmittelbar ausgeübt oder der Staat wird gezwungen zu reagieren - als imperative Partizipation bezeichnet werden soll, kann nun in einem nächsten Schritt das Augenmerk auf die Funktionen von Partizipation gelenkt werden.

\section{B. Funktionen von Partizipation: Demokratie, Rechtsstaatlichkeit, Effektivität}

In dieser Definition klingt schon an, dass Partizipation eng mit Demokratie- und Rechtsstaatsprinzip verbunden ist: Wahlen und Abstimmungen sind dem Demokratieprinzip zugehörig, eine rechtsförmige Reaktion des Staates durch die Verwaltung oder die Gerichte ist primär dem Rechtsstaatsprinzip - das hier als materielles Rechtsstaatsprinzip und damit als den Schutz der Grundrechte mitumfassend verstanden wird - zuzuordnen. Dementsprechend sind die Funktionen von Partizipation mehr der rechtsstaatlichen Sphäre oder mehr der demokratischen Sphäre zugehörig. Als dritte Funktion kommt die Effektivierung staatlicher Gewalt hinzu.

Zunächst zur rechtsstaatlichen Sphäre: Dem Schutz von Rechtsstaatlichkeit und Grundrechten dient Partizipation, weil und wenn sie auf die Sicherung individueller Rechte gerichtet ist. Seit der Mühlheim-KärlichEntscheidung des Bundesverfassungsgerichts von 1979 ist anerkannt, dass auch der Schutz individueller Rechte eine der Funktionen von Beteiligung ist. ${ }^{10}$ So werden als Funktion von Partizipation rechtsstaatliche Aspekte wie ein (präventiver) vorhabenbezogener Grundrechtsschutz genannt, ${ }^{11}$

9 BVerfGE 69, 315 - Brokdorf, LS 1: „[Die Versammlungsfreiheit] gehört zu den unentbehrlichen Funktionselementen eines demokratischen Gemeinwesens.".

10 BVerfGE 53, 30 (65 f.); siehe dazu ausführlich A. Fisahn, Demokratie und Öffentlichkeitsbeteiligung, 2002, $200 \mathrm{ff}$.

11 I. Appel, Staat und Bürger im Umweltverwaltungsverfahren, NVwZ 31 (2012) 1361 (1362); A. von Bogdandy, Gubernative Rechtsetzung, 2000, 68; E. Gurlit, 
ebenso die Gewährleistung eines fairen Verfahrens ${ }^{12}$ oder die Herstellung von Waffengleichheit. ${ }^{13}$ Diese Funktionen werden der Verwaltung zugeordnet, werden aber auch gleichermaßen auf Ebene der Judikative verwirklicht. Partizipation bezweckt also die Wahrung individueller Rechte und damit von Rechtsstaatlichkeit und Grundrechten. Partizipation dient damit der Sicherung individueller Selbstbestimmung, die in Art. 1 Abs. 1 GG, der Menschenwürdegarantie, ihren entscheidenden Ausdruck findet. ${ }^{14}$

Partizipation dient aber auch der Demokratie. Dies ist ihre zweite Funktion. Wahlen und Abstimmungen sind primär demokratisch fundiert. Dies zeigt schon Art. 20 Abs. 2 S. 1 GG, der alle Staatsgewalt vom Volk ausgehen lässt. Auch das Bundesverfassungsgericht betont, dass Demokratie die „freie Selbstbestimmung aller Bürger"15 gewährleiste. Ebenso lässt sich auf Ebene des Verwaltungsrechts Beteiligung demokratisch verstehen. Ganz allgemein wird die Demokratisierung von Entscheidungen als Zweck von Beteiligung genannt. ${ }^{16}$

Für diese demokratische Begründung - die keineswegs unumstritten ist $^{17}$ - lässt sich anführen, dass durch Partizipation die Staatsgewalt in einem offenen Prozess an die Gesellschaft rückgebunden wird. ${ }^{18}$ Durch Deliberation ${ }^{19}$ wird neben der Rückbindung der Staatsgewalt an die Bürger auch ein Interessenausgleich ${ }^{20}$ ermöglicht: der Verantwortungszusam-

Neue Formen der Bürgerbeteiligung? Planung und Zulassung von Projekten in der parlamentarischen Demokratie, JZ 67 (2012), 833 (834).

12 J. Stender-Vorwachs, Neue Formen der Bürgerbeteiligung?, NVwZ 31 (2012), 1061 (1063); J. Ziekow, Neue Formen der Bürgerbeteiligung? Planung und Zulassung von Projekten in der parlamentarischen Demokratie, Gutachten D zum 69. Deutschen Juristentag, 2012, 15.

13 J. Stender-Vorwachs (Fn. 12), 1063; I. Appel (Fn. 11), 1362; J. Ziekow (Fn. 12), 15.

14 Vgl. A. von Bogdandy (Fn. 11), 31; A. Wallrabenstein, Das Verfassungsrecht der Staatsangehörigkeit, 1999, 91, 99.

15 BVerfGE 44, 125 (142).

16 I. Appel (Fn. 11), 1362.

17 Vgl. ausführlich A. Fisahn (Fn. 10), $198 \mathrm{ff}$.

18 A. Fisahn (Fn. 10), 334. Auch das Bundesverfassungsgericht stellt in seinem Demokrativerständnis stark auf die Kommunikationsbeziehung zwischen Bürgern und Staat ab, siehe zuletzt BVerfGE 134, 141, LS 1.

19 A. Fisahn, Abgeleitete Demokratie, KritV 79 (1996), 267, (278 ff.); vgl. J. Habermas, Faktizität und Geltung, 1992, 151 ff., 349 ff.; N. Petersen, Demokratie und Grundgesetz, MPI Preprint 2008, 28 ff.; H. Heinelt, Governing Modern Societies. Towards Participatory Governance, 2010, 8.

20 Vgl. A. Fisahn (Fn. 10), $213 \mathrm{ff}$. 
menhang zwischen Staatsgewalt und Gesellschaft wird gestärkt, indem der Staat den Bürgern antworten muss. Dieser Rückbindung dienen zwar grundsätzlich Wahlen. Wahlen finden aber nur alle vier Jahre statt, weshalb die Rückbindung relativ schwach ist. ${ }^{21}$ Deshalb betont das Bundesverfassungsgericht, dass die Bürger auch zwischen den Wahlen das Staatshandeln beeinflussen können müssen. ${ }^{22}$ Beteiligung dient außerdem auch der (demokratischen) Kontrolle des Staates durch die Bürger. ${ }^{23}$ Voraussetzung dafür ist die Schaffung von Transparenz, die auch demokratisch fundiert ist. ${ }^{24}$ Auch die Erwägungsgründe zum Übereinkommen über den $\mathrm{Zu}$ gang zu Informationen, die Öffentlichkeitsbeteiligung an Entscheidungsverfahren und den Zugang zu Gerichten in Umweltangelegenheiten (Aarhus-Konvention), ${ }^{25}$ die in Deutschland durch das Umweltinformationsgesetz, das Öffentlichkeitsbeteiligungsgesetz und das Umweltrechtsbehelfsgesetz umgesetzt wurde, zeigen, dass Beteiligung demokratisch zu verstehen ist. Dort heißt es, dass ,die Durchführung dieses Übereinkommens zur Stärkung der Demokratie [...] beitragen wird“. Schließlich soll durch Partizipation die Akzeptanz bestimmter Vorhaben in der Öffentlichkeit gesteigert werden. ${ }^{26}$ Dies erfüllt ebenfalls einen demokratischen Zweck ${ }^{27}$ - da es um die Rückbindung des Staates an den Einzelnen geht -, weist gleichzeitig aber auch schon in Richtung Effektivität, da Akzeptanz Klagen und Proteste zu verhindern weiß.

Die Effektivierungsfunktion ist die dritte Funktion staatlichen Handelns. Sie wird erfüllt durch die Informierung der Behörde - damit diese bessere und „richtigere“ Entscheidungen treffen kann,$-{ }^{28}$ die Vermeidung

21 A. von Bogdandy (Fn. 11), $70 \mathrm{ff}$.

22 BVerfGE 20, 56 (98).

23 T. Groß (Fn. 1), 511; E. Gurlit (Fn. 11), 835; I. Appel (Fn. 11), 1362; U. Battis, Partizipation im Städtebaurecht, 1976, $198 \mathrm{ff}$.

24 J. Bröhmer, Transparenz als Verfassungsprinzip, 2004, 41 ff.; S. Smith, Konfliktlösung im demokratischen Bundesstaat, 2011, 351.

25 Aarhus-Konvention vom 25. Juni 1998, BGB1 II 2006, 1252.

26 Siehe nur C. Calliess/M. Dross, Neue Netze braucht das Land: Zur Neukonzeption von Energiewirtschaftsgesetz und Netzausbaubeschleunigungsgesetz, JZ 67 (2012), 1002 (1003, 1010 f.); I. Appel (Fn. 11), 1362.

27 Ebenso E. Schmidt-Aßmann, Verwaltungsorganisationsrecht als Steuerungsressource, Einleitende Problemskizze, in: W. Hoffmann-Riem/E. Schmidt-Aßmann (Hrsg.), Verwaltungsorganisationsrecht als Steuerungsressource, 5 (39), der aber keine weitere Erklärung liefert.

28 BVerfGE 33, 125 (159); E. Gurlit (Fn. 11), 834; A. von Bogdandy (Fn. 11), 68 f.; I. Appel (Fn. 11), 1362. 
von Rechtsstreitigkeiten ${ }^{29}$ und schliesslich die Beschleunigung des Verfahrens. ${ }^{30}$

Es lässt sich also festhalten: Funktionen von Partizipation sind die Effektivierung staatlichen Handelns, der Schutz individueller Rechte und die Ermöglichung und Förderung von Demokratie. Diese Funktionen stehen aber in einem Spannungsverhältnis zueinander. Dies wird an folgendem Beispiel deutlich: Wenn die Anwohner entscheiden dürften, ob Stromleitungen gebaut werden dürften, dann gäbe es keine Stromleitungen, denn alle sagen „NIMBY - Not in my backyard!“ Eine solche Betroffenenentscheidung widerspräche der (effektiven Durchsetzung der) demokratischen Entscheidung für die Energiewende, denn diese wird von fast allen Bürgern gewünscht, bloß eben nicht im eigenen „Hintergarten.“ Eine Betroffenenentscheidung widerspräche u.U. auch den Grundrechten des Projektträgers, also des Betreibers der Stromleitungen. Wenn die Anwohner aber gar nichts zu sagen hätten, dann wären ihre Grundrechte und ebenso ihre Interessen bedeutungslos. Widerspruch und Widerstand wären die Folge, eine effektive Durchsetzung der Entscheidung somit kaum durchführbar. Das Spannungsverhältnis muss daher so aufgelöst werden, dass Konflikte zwischen Rechtsstaatlichkeit und Demokratie ausbalanciert werden. Dafür muss die Frage beantwortet werden, wer mit welchem Einfluss partizipieren darf.

\section{Die Gewaltenteilung des Grundgesetzes: Art. 20 Abs. 2 S. 1 GG und Abs. 3 GG}

Der Schlüssel zur Konzeptualisierung von Partizipation liegt im Gewaltenteilungsgrundsatz: Er betrifft ebenso wie Partizipation das Innehaben und die Teilhabe an der Ausübung von Staatsgewalt und verfolgt ebenso wie Partizipation eine demokratische, eine rechtsstaatliche und eine Effektivitätsfunktion. Da der Gewaltenteilungsgrundsatz diese widerstreitenden Funktionen ausbalanciert, kann er auch Partizipation steuern und ausgestalten.

29 I. Appel (Fn. 11), 1362.

30 E. Röper, Zwischenruf: Von der Petition zum Runden Tisch, ZRP 45 (2012), 25 (27): Bürgerbeteiligung als „Motor statt Bremse“; siehe auch J. Ziekow/M. P. Oertel/A. Windoffer, Dauer von Zulassungsverfahren, 2005, $121 \mathrm{ff}$., $211 \mathrm{ff}$.; a.A. W. Hertel/C.-D. Munding (Fn. 3), $2624 \mathrm{f}$. 
Erstens, der Gewaltenteilungsgrundsatz schützt Rechtsstaatlichkeit und individuelle Freiheit, indem er die Ausübung öffentlicher Gewalt verschiedenen Organen zuweist und so die Staatsgewalt hemmt, mäßigt und kontrolliert. ${ }^{31}$

Zweitens, der Gewaltenteilungsgrundsatz mäßigt nicht nur die Ausübung öffentlicher Gewalt, sondern dient auch dazu, sie zu effektivieren: Die Staatsgewalt wird nämlich nicht nur verschiedenen Organen zugewiesen, sondern auch und gerade den Organen, „die dafür nach ihrer Organisation, Zusammensetzung, Funktion und Verfahrensweise über die besten Voraussetzungen verfügen.“ 32 Die Gewaltenteilung wird so als „Funktionenordnung “33 verstanden. Es geht um die „Gewährleistung effektiver Funktionalität", 34 also die Herstellung von „Funktionsadäquanz“"35 oder gar „Funktionsgerechtigkeit.“36 Diese Funktion steht heute im Vorder-

31 Vgl. nur BVerfGE 34, 52 (59); 68, 1 (86); 95, 1 (15); B. Grzeszick, Art. 20 (Gewaltenteilung), Rn. 29 ff., in: T. Maunz/G. Dürig, Grundgesetzkommentar, Loseblattsammlung Band III, 70. Lieferung Dez. 2013.

32 BVerfGE 68, 1 (86); 95, 1 (15); 98, 218 (251 f.).

33 N. Achterberg, Probleme der Funktionenlehre, 1970, 112; G. Zimmer, Funktion Kompetenz - Legitimation, Gewaltenteilung in der Ordnung des Grundgesetzes, 1979, 55; C. Möllers, Gewaltengliederung, 2005, 3; R. Thoma, Die Funktionen der Staatsgewalt, in: G. Anschütz/R. Thoma (Hrsg.), Handbuch des Deutschen Staatsrechts, Band II, 1932, 108 (113) spricht von Funktionenteilung; G. Jellinek, Allgemeine Staatslehre, 3. Auflage 1912, 595 ff., von Funktionenlehre und M. Jestaedt, Demokratieprinzip und Kondominialverwaltung, 1993, S. 168 von Funktionengliederung.

34 T. von Danwitz, Der Grundsatz funktionsgerechter Organstruktur, Der Staat 35 (1996), 329 (345); W. R. Wrege, Das System der Gewaltenteilung im Grundgesetz, Jura 1996, 436 (436).

35 B. Grzeszick (Fn. 31), Art. 20 (Gewaltenteilung), Rn. 51; T. von Danwitz (Fn. 34), 329 ff. Dazu nur W. Leisner, Die quantitative Gewaltenteilung, DÖV 1969, 405 (407 ff.); R. Poscher, Funktionenordnung des Grundgesetzes, in: W. HoffmannRiem/E. Schmidt-Aßmann/A. Voßkuhle (Hrsg.), Grundlagen des Verwaltungsrechts Band I, 2. Aufl. 2013, 543 (557): „funktionsadäquate Aufgabenwahrnehmung" mit jeweils weiteren Nachweisen.

36 H. D. Horn, Gewaltenteilige Demokratie, demokratische Gewaltenteilung: Überlegungen zu einer Organisationsmaxime des Verfassungsstaates, Archiv des öffentlichen Rechts 127 (2002), 427 (448 mwN.); M. Cornils, Gewaltenteilung, in: O. Depenheuer/C. Grabenwarter (Hrsg.), Verfassungstheorie, 2010, 657 (683 ff.); P. Lerche, Gewaltenteilung - deutsche Sicht, in: J. Isensee (Hrsg.), Gewaltenteilung heute, 2000, 75 (75) spricht ebenso wie O. Küster, Das Gewaltenproblem im modernen Staat, Archiv des öffentlichen Rechts 75 (1949), 397 (402) und T. von Danwitz (Fn. 34), von ,funktionsgerechter Organstruktur“. 
grund. ${ }^{37}$ Damit muss dem Organ die Aufgabe zugewiesen sein, die ihm sowohl nach Struktur wie auch nach Legitimation entspricht, ${ }^{38}$ damit es effizient entscheiden kann. ${ }^{39}$

Drittens, aus der zweiten Funktion ergibt sich, dass Organe geschaffen und mit der Kompetenz ausgestattet werden müssen, Staatsgewalt auszuüben. Politik konstituiert sich nämlich erst durch rechtsförmige Organe ${ }^{40}$ und mit einer Kompetenzzuweisung wird immer auch Entscheidungsgewalt und Entscheidungsfähigkeit eingeräumt. ${ }^{41} \mathrm{Da}$ die Ausübung von Staatsgewalt unter dem Grundgesetz immer auf das Volk zurückgeführt werden muss, folgt daraus zwingenderweise die demokratische Funktion der Gewaltenteilung. ${ }^{42}$ Das Ziel der Gewaltenteilungslehre ,ist die parlamentarisch-demokratische Konstituierung, Legitimierung und Steuerung der Staatsmacht [...] Damit präsentiert sich Gewaltenteilung [...] als Modus der demokratischen Ordnung. “43

Viertens, der Gewaltenteilungsgrundsatz löst die Konflikte zwischen Rechtsstaatlichkeit und Demokratie auf - und kann daher auch für die Ausgestaltung von Partizipation fruchtbar gemacht werden. Ein neues Verständnis von Gewaltenteilung, entwickelt von Christoph Möllers in seiner Habilitation „Gewaltengliederung“, stellt für die Auflösung des Konflikts zwischen Demokratie und Rechtsstaatlichkeit auf folgenden Gedanken-

37 O. Küster (Fn. 36), 402; W. R. Wrege (Fn. ), 440. Anders aber S. Huster/J. Rux, Art. 20 GG, Rn. 156, in: C. Hillgruber/V. Epping (Hrsg.), Grundgesetz. Kommentar, 2. Auflage 2013.

38 H. D. Horn (Fn. 36), 447 f.

39 F. Ossenbühl, Aktuelle Probleme der Gewaltenteilung, DÖV 1980, 545 (549).

40 Vgl. B. Grzeszick (Fn. 31), Art. 20 (Gewaltenteilung), Rn. 57 f. S. auch H. Seiler, Gewaltenteilung: Allgemeine Grundlagen und schweizerische Ausgestaltung, 1994, $238 \mathrm{ff}$.

41 T. von Danwitz (Fn. 34), 335, der hier dementsprechend auch den Rückbezug zum demokratischen Prinzip herstellt.

42 C. Möllers (Fn. 33), 3; H. D. Horn, Die grundrechtsunmittelbare Verwaltung, 1999, 261 ff.; F. Ossenbühl, Schlußwort, in: J. Isensee (Hrsg.), Gewaltenteilung heute, 2000, 123 (127); E. Schmidt-Aßmann, Das allgemeine Verwaltungsrecht als Ordnungsidee, 2. Aufl. 2004, 179 ff., M. Jestaedt (Fn. 33), 168 f.; ähnlich $B$. Grzeszick (Fn. 31), Art. 20 (Gewaltenteilung), Rn. 75, der „Gewaltenteilung als organisatorische[n] Ausdruck und Verstärkung von Demokratie und Rechtsstaat“ versteht; siehe auch U. Di Fabio, Gewaltenteilung, in: J. Isensee/P. Kirchhof (Hrsg.), Handbuch des Staatsrechts Band II, 3. Auflage 2004, Rn. 9, der darauf verweist, dass die Rechtsprechung immer wieder das Parlament anhält, seiner Verantwortung gerecht zu werden.

43 H. D. Horn (Fn. 36), 540 f. 
gang ab: Entscheidungen, die in vornehmlich demokratisch fundierten Verfahren zustande kommen, weisen bestimmte Eigenschaften auf, die fundamental anders sind als Eigenschaften von Entscheidungen, die in Verfahren zum Schutz von Rechtsstaatlichkeit und Grundrechten getroffen werden. Es geht dabei um die „Intensität der Verrechtlichung“ der Entscheidung, ihre „Zeitliche Orientierung“ und ihre „Reichweite“. ${ }^{44}$ Demokratie verwirklicht sich vornehmlich durch Entscheidungen, die rechtlich selbst wenig determiniert sind, die zukunftsgerichtet sind und die die Allgemeinheit betreffen. Rechtsstaatlichkeit und Grundrechte werden vornehmlich geschützt durch Entscheidungen, die rechtlich selbst strikt determiniert sind, in der Vergangenheit liegende Sachverhalte betreffen und nur Einzelne binden.

Daraus folgt, dass jede staatliche Entscheidung über den Gewaltenteilungsgrundsatz mit einer der drei Gewalten verknüpft ist: Entscheidungen in demokratischen Verfahren werden grundsätzlich von der Legislative getroffen. Eine solche Entscheidung, d.h. ein Parlamentsgesetz, zeichnet sich dadurch aus, dass es allein durch die Verfassung rechtlich determiniert ist, zukünftige Sachverhalte regelt und die Allgemeinheit betrifft. ${ }^{45}$ Entscheidungen in Verfahren zum Schutz von Rechtsstaatlichkeit und Grundrechten werden grundsätzlich von der Judikative getroffen: eine solche Entscheidung, also ein Gerichtsurteil, ist durch das gesamte Gesetzesrecht determiniert, hat einen in der Vergangenheit liegenden Sachverhalt zum Gegenstand und betrifft nur den Kläger sowie mögliche Verfahrensbeteiligte. ${ }^{46}$ Die Exekutive steht zwischen beiden Polen. ${ }^{47}$ Hier wird der Bogen zwischen Demokratie und Rechtsstaatlichkeit besonders deutlich: Auf der einen Seite steht als „quasi-legislatorische“ Gewalt die Regierung und auf der anderen Seite als „quasi-judikative“ Gewalt eine untere Behörde. Die Regierung kann ähnlich einem Parlament (materielle) Gesetze erlassen,

44 C. Möllers (Fn. 33), 17; C. Möllers, Dogmatik der grundgesetzlichen Gewaltengliederung, Archiv des öffentlichen Rechts 132 (2007), 493 (508).

45 C. Möllers (Fn. 33), 58, 105 ff.; C. Möllers (Fn. 44), 509 ff.; s. auch BVerfGE, 135, 1 (Rn. 55).

46 C. Möllers (Fn. 33), 58, 95 ff.; C. Möllers (Fn. 44), 512 ff.; s. auch K. F. Gärditz, Entwicklungen und Entwicklungsperspektiven des Verwaltungsprozessrechts zwischen konstitutioneller Beharrung und unionsrechtlicher Dynamisierung, Die Verwaltung 46 (2013), 257, 264 f.; C. Franzius, Möglichkeiten und Grenzen der richterlichen Rechtsfortbildung zur Bestimmung der Klagebefugnis im Umweltrecht, DVB1. 2014, 543 (549).

47 C. Möllers (Fn. 33), 112 ff.; C. Möllers (Fn. 44), 514 ff. 
diese sind dabei aber rechtlich vordeterminierter, da sie im Rahmen des gesetzlichen Ermächtigungsspielraums bleiben müssen. Die unteren Behörden entscheiden ähnlich wie Gerichte. Ihnen kommt aber, z.B. in Ermessensfällen, ein größerer Entscheidungsspielraum als den Gerichten zu. $\mathrm{Zu}$ beachten ist, dass aus dieser Zuordnung zwar grundsätzliche Linien entwickelt werden können, aber keine kategorischen Schlussfolgerungen zu ziehen sind. So betreffen Gerichtsentscheidungen auch immer die $\mathrm{Zu}$ kunft und Gesetze können auch die Vergangenheit regeln. Verfahren, in denen Entscheidungen zum Schutz von Rechtsstaatlichkeit und Grundrechten getroffen werden, sind immer auch demokratisch legitimiert. Auch Verfahren, die kollektive Selbstbestimmung ermöglichen, müssen Grundrechte achten. Dies zeigt schon Art. 1 Abs. 3 GG, der alle Staatsgewalt an die Grundrechte bindet.

Nach diesem Modell schlägt die Gewaltenteilung durch die Zuordnung von bestimmten Entscheidungen zu bestimmten Gewalten einen Bogen zwischen Demokratie und Rechtsstaatlichkeit und balanciert so die Konflikte zwischen beiden Prinzipien aus.

\section{Der Gewaltenteilungsgrundsatz als Mittel zur Konzeptualisierung von Partizipation}

Überträgt man diesen Gedankengang auf Partizipation - denkt man also von den verschiedenen Gewalten, ihren Funktionen und der entsprechenden Zuordnung bestimmter Entscheidungen zu einer Gewalt aus -, so ist die entscheidende Frage, ob Partizipation in einem bestimmten Fall mehr dem Demokratieprinzip oder mehr dem Rechtsstaatsprinzip zugehörig ist. Dementsprechend richtet sich die Partizipationsform nach der jeweiligen Gewalt. Partizipationsformen, die primär einer demokratischen Funktion folgen, knüpfen an Handlungen der Legislative an. Partizipationsformen, die primär eine rechtsstaatliche und damit grundrechtsschützende Funktion verfolgen, knüpfen an Handlungen der Judikative an. Partizipationsformen, die an Handlungen der Exekutive anknüpfen, variieren zwischen beiden Polen.

Die Eigenschaften der jeweiligen Partizipationsform müssen so ausgestaltet sein, dass sie Konflikte zwischen Demokratie und Rechtsstaatlichkeit ausbalancieren. Ansonsten läge ein Verstoß gegen den Gewaltenteilungsgrundsatz vor. Diese Eigenschaften sind der Kreis der Beteiligten und der Grad ihres Einflusses auf die Entscheidung und werden im Fol- 
genden anhand des deutschen Rechts entfaltet. So kann ermittelt werden, wie Partizipation im Einzelnen nach dem Grundgesetz beschaffen sein muss - und größtenteils schon beschaffen ist.

I. Die Judikative: Schutz von Rechtsstaatlichkeit und Grundrechten durch Partizipation

Partizipation auf Judikativebene muss so ausgestaltet sein, dass sie Rechtsstaatlichkeit und Grundrechte schützt. Dafür bedarf es eines Verfahrens, das den in seinen Rechten betroffenen Einzelnen in den Mittelpunkt stellt - um dessen Rechte geht es zunächst. Der Einzelne leitet dementsprechend das Verfahren ein, die Letztentscheidungsmacht verbleibt jedoch bei Gericht. Könnte der Kläger etwa Entscheidungen zum unmittelbaren Nachteil der anderen treffen, z.B. selbst das Urteil sprechen, so wären die Rechte anderer Rechtsbetroffener nicht gesichert. Dies wäre Fremd-, nicht Selbstbestimmung. Die Entscheidungshoheit des Gerichts dient zum anderen dem Schutz demokratischer Entscheidungen. Nur wenn im Rahmen der Gesetze entschieden wird, wird die demokratische Entscheidung - und damit auch die formale Gleichheit aller - geachtet.

Das geltende Recht entspricht dem vorher Gesagten: Um individuelle Selbstbestimmung und damit auch individuelle Legitimation zu stärken, wirkt der Einzelne in gerichtlichen Verfahren mit, indem er u.a. das Verfahren einleitet, ${ }^{48}$ den Streitgegenstand bestimmt ${ }^{49}$ und das Verfahren beispielsweise durch eine Klagerücknahme ${ }^{50}$ auch wieder beenden kann. Diese Entscheidungsmacht stellt keine Fremdbestimmung der Gegenseite dar, da sie jene nicht benachteiligt. Unterhalb der Ebene der Letztentscheidung kann der Einzelne damit sogar selbst Entscheidungen treffen, solange sie seine individuelle Selbstbestimmung fördern, die der anderen nicht einschränken und im Rahmen der Gesetze bleiben, die im Wege kollektiver

$48 \S 81 \mathrm{VwGO}$ § $253 \mathrm{ZPO}$.

$49 \S 82$ VwGO i.V.m. § 88 VwGO; § 253 ZPO.

$50 § 92$ VwGO; § 269 ZPO. Nach Stellung der Anträge bzw. nach Beginn der mündlichen Verhandlung ist dies aber nur mit Einwilligung des Beklagten möglich, $\S 92$ Abs. 1 S. 2 VwGO bzw. § 269 Abs. 2 ZPO. 
Selbstbestimmung erlassen wurden. Das Urteil, also die Entscheidung, auf die es letztendlich ankommt, erlässt allein das staatliche Gericht. ${ }^{51}$

\section{Die Legislative: Ermöglichung von Demokratie durch Partizipation}

Partizipation auf Legislativebene muss Demokratie fördern. Dementsprechend kann das ganze Volk für das ganze Volk entscheiden. Die Zugehörigkeit zum Kollektiv, nicht die materielle Rechtsbetroffenheit löst die Beteiligungsrechte aus. Das Volk entscheidet über die Zusammensetzung des Parlaments. In der repräsentativen Demokratie hat das Volk allerdings einen nur sehr beschränkten Einfluss auf Sachfragen.

Volksgesetzgebung existiert in der Schweiz, ${ }^{52}$ eingeschränkt in Österreich ${ }^{53}$ sowie in allen deutschen Bundesländern. ${ }^{54}$ Dass es auf Bundesebene in Deutschland keine Volksgesetzgebung gibt, stellt ein Defizit dar. ${ }^{55}$

Genauso wie auf Judikativebene sind auch auf Legislativebene Abweichungen durch Beteiligungsformen ohne Entscheidungsmacht, etwa Anhörungsrechte, möglich. Das Volk kann dementsprechend auch Partizipationsmöglichkeiten unterhalb der Entscheidungsebene innehaben. Dies fördert Demokratie, sofern potentiell alle beteiligt werden. In Deutschland fehlen solche Mitwirkungsmöglichkeiten fast vollständig: In den Bundestagsausschüssen finden nach $\S 70$ der Geschäftsordnung des Bundestages nur Anhörungen von Experten und Interessensvertretern statt. Das Volk wird nicht gefragt. Dies ist in anderen Staaten anders, z.B. in Südafrika. Hier wurden schon Gesetze vom Verfassungsgericht für nichtig erklärt,

$51 \S 107$ VwGO, § 300 ZPO. Die Mitwirkung von Privaten, nämlich Schöffen, stellt in diesem Kontext kein Problem dar, da diese weder aktuell noch potentiell in eigener Sache entscheiden. Amicus-Curiae-Briefs fallen aus dem oben dargestellten Partizipationsbegriff heraus, da sie lediglich eine Anregung an das Gericht darstellen und von diesem nicht beachtet werden müssen.

52 Vgl. dazu R. Rhinow, Grundprobleme der schweizerischen Demokratie, Zeitschrift für Schweizerisches Recht 103 (1984), 116; A. Vatter, Das politische System der Schweiz, 2014, $343 \mathrm{ff}$.

53 S. P. Bußjäger/A. Balthasar/N. Sonntag (Hrsg.), Direkte Demokratie im Diskurs: Beiträge zur Reform der Demokratie in Österreich, 2014.

54 Siehe dazu die sehr gute Übersicht bei J. Rux (Fn. 4) und P. Neumann (Fn. 4).

55 H. Dreier/F. Wittreck, Repräsentative und direkte Demokratie im Grundgesetz, in: L. Feld/P. M. Huber/O. Jung/C. Welzel/F. Wittreck (Hrsg.), Jahrbuch Direkte Demokratie 2009, 11-39. Vgl. auch die Hinweise in Fn. 4. 
weil die Bürger vorab nicht beteiligt wurden. ${ }^{56}$ Auch in Baden-Württemberg verfügen die Bürger über Möglichkeiten die Gesetzgebung zu beeinflussen. Sie werden eingeladen, ihre Meinungen online in den Gesetzgebungsprozess einzubringen. ${ }^{57}$ Diese Beiträge werden gelesen und bewertet. Sie können im Gesetz ebenso Berücksichtigung finden wie Expertenmeinungen - und dies ist nach Eigenauskunft der Staatsregierung auch schon geschehen. ${ }^{58}$ In entsprechender Weise sollte auf Bundesebene Legitimation von Staatsgewalt gestärkt werden.

Partizipation an der Gesetzgebung dient der Ermöglichung von Demokratie. Dafür muss Partizipation durch Wahlen und Abstimmungen allen Bürgern offen stehen. Auch unterhalb der Schwelle, Entscheidungen zu treffen, fördert Partizipation die Demokratie.

\section{Die Exekutive: Partizipation im Spannungsfeld von Demokratie und Rechtsstaatlichkeit}

Die Exekutive wird ganz unterschiedlich tätig, in ihr sind rechtsstaatliche und demokratische Elemente eng miteinander verwoben. ${ }^{59}$ Dennoch gibt es meist einen Vorrang des einen oder des anderen Prinzips. Welches Prinzip im Vordergrund steht, richtet sich nach den drei oben genannten Eigenschaften „zeitliche Orientierung“, „Intensität der Verrechtlichung“ und „Reichweite der Entscheidung“. Die Partizipationsform orientiert sich daran, ob im Einzelfall das rechtsstaatliche Prinzip oder das demokratische

56 South African Constitutional Court, Doctors for Life International v the Speaker of the National Assembly and Others, 2006 (6) SA 416 (CC); South African Constitutional Court, Matatiele Municipality and Others $v$ President of the Republic of South Africa and Others, 2007 (1) BCLR 47 (CC). Siehe dazu T. Roux, The Principle of Democracy in South African Constitutional Law, in: S Woolman/M. Bishop (Hrsg.), Constitutional Conversations, 2008, 79 und D. Brand, Reply: Writing the Law Democratically, in: ebd., 97-111. S. dazu auch den Beitrag von B. Loots, Civic Dignity as the Basis for Public Participation in the Legislative Process, in diesem Band.

57 Siehe www.beteiligungsportal.baden-wuerttemberg.de (zuletzt aufgerufen am 5. Februar 2015). S. dazu den Beitrag von F. Reidinger, Baden-Württemberg zwischen Wählen, Mitreden und Entscheiden - Mehr Partizipation als Regierungsauftrag, in diesem Band.

58 www.beteiligungsportal.baden-wuerttemberg.de/de/informieren/service/pressemitt eilung/pid/mitgestalten-von-zu-hause-aus (zuletzt aufgerufen am 5. Februar 2015).

59 C. Möllers (Fn. 33), 112 ff.; C. Möllers (Fn. 44), 514 ff. 
Prinzip im Vordergrund steht. Im Folgenden wird zunächst die Regierung, die Rechtsverordnungen erlässt, behandelt (1.), dann die Planfeststellungsbehörden (2.) und zuletzt die Ordnungsbehörden (3.). Diese drei Organe schlagen beispielhaft den Bogen innerhalb der Exekutive von mehr Demokratie hin zu mehr Rechtsstaatlichkeit. Dass Partizipation nicht nur rechtsstaatlich, sondern auch demokratisch zu verstehen ist, wird an den beiden ersten Beispielen besonders deutlich.

\section{Die Regierung / Rechtsverordnungen}

Auf der einen Seite des Bogens steht die Regierung. Sie ist Teil der Exekutive, ${ }^{60}$ wird aber auch legislativ durch Rechtsverordnungen tätig. Hier liegt der Schwerpunkt auf der Ermöglichung demokratischer Entscheidungen.

\section{a. Keine Entscheidungsgewalt des Volkes}

Partizipation dient hier ebenso wie bei Wahlen und Abstimmungen v.a. der Förderung und Ermöglichung von Demokratie. Anders als auf Legislativebene steht dem Volk aber keine Entscheidungsgewalt zu. Im Gegensatz zum Parlament, das vom Volk in Fällen direkter Demokratie ersetzt wird, ist eine Ersetzung der Regierung auf Exekutivebene nicht möglich. Dies liegt daran, dass nur auf Legislativebene Demokratie voll verwirklicht wird und daher nur hier der Demos auch entscheiden darf. Die Regierung ist nicht parlamentsgleich, wie schon Art. 20 Abs. 2 S. 2 GG zeigt, der von den besonderen Organen der Gesetzgebung, der vollziehenden Gewalt und der Rechtsprechung spricht. Auch die Gesetzgebungsvorschriften des Grundgesetzes und hier vor allem Art. 80 GG verdeutlichen, dass die Regierung zwar materielle Gesetze erlassen darf, diese aber anderen und strikteren Regeln unterliegen als formelle Parlamentsgesetze. ${ }^{61}$ Die Legis-

60 Die Verwaltung erfasst auch die Regierungsfunktion, siehe W. Krebs, Art. 50, Rn. 11, in: I. von Münch/P. Kunig (Hrsg.) Grundgesetz. Kommentar, Band 1, 6. Auflage 2012; O. Dörr, Art. 50, Rn 19, in: C. Hillgruber/V. Epping (Hrsg.), Grundgesetz. Kommentar, 2. Auflage 2013.

61 Siehe dazu F. Ossenbühl, Rechtsverordnung (§ 103), in: J. Isensee/P. Kirchhof (Hrsg.), Handbuch des Staatsrechts, Band V, 3. Auflage 2007. 
lativtätigkeit der Regierung ist folglich stärker rechtlich gebunden als die des Parlaments. Auf der oben aufgezeigten Skala bewegt sie sich schon ein Stück weg von der vollen Verwirklichung demokratischer Entscheidungen.

\section{b. Weitere Einflussnahmemöglichkeiten}

Trotzdem bleibt weiterhin eine Einflussnahme durch Partizipation am Regierungshandeln möglich. Diese Einflussnahme kann auf zwei verschiedenen Ebenen erfolgen: Zum einen binden formelle Gesetze die Regierung. Diese können de lege lata auf Landesebene und de lege ferenda auf Bundesebene auch im Wege direkter Demokratie zustande kommen. So kann das Volk durch seine Entscheidung die Regierung und deren Rechtsverordnungsgebung steuern. Dies muss nach den allgemeinen Regeln des Grundgesetzes geschehen, etwa nach Art. 80 GG und dem Grundsatz der Gewaltenteilung. Zum anderen sind beim Erlass von Rechtsverordnungen auch Beteiligungsformen unterhalb der Entscheidungsebene denkbar. Zwar sieht Art. 80 GG keine entsprechenden Regelungen vor. Jedoch finden sich entsprechende Vorschriften in der Gemeinsamen Geschäftsordnung der Bundesministerien (GGO), in den ermächtigenden Gesetzen und im Raumordnungsgesetz (ROG).

\section{(1) Gemeinsame Geschäftsordnung der Bundesministerien}

Zentral- und Gesamtverbände sind nach $\S 62$ Abs. 2 i.V.m. $\S 47$ Abs. 3 GGO in die Rechtsverordnungsgebung einzubinden. Zeitpunkt, Umfang und Einfluss der Beteiligung sowie die Auswahl der Beteiligten bleiben aber nach $\S 47$ Abs. 3 S. 2 GGO dem Ermessen des federführenden Bundesministeriums überlassen. Außerdem ist die Norm eine des Innenrechts, ${ }^{62}$ sodass sich die zu Beteiligenden nicht auf sie berufen können. ${ }^{63}$ Hier existieren also keine Partizipationsrechte des Einzelnen. Stattdessen

62 V. Epping, Art. 65 GG, Rn. 19.3, in: C. Hillgruber/V. Epping (Hrsg.), Grundgesetz Kommentar, 2. Auflage 2013: „Verwaltungsvorschrift“.

63 Das Gleiche gilt auch für Gesetzesinitiativen. Dies ergibt sich aus $\S 47$ Abs. 3 GGO II ohne den Verweis aus $§ 62$ Abs. 2 GGO II. 
besteht eine rudimentäre Verpflichtung, Partizipation durch Verbände zu ermöglichen. ${ }^{64}$

(2) Vorschriften in den ermächtigenden Gesetzen

Für spezielle Rechtsverordnungen sehen manche der zu ihrem Erlass ermächtigenden Gesetze vor, dass sogenannte „beteiligte Kreise“ zu hören seien. ${ }^{65}$ Nach $\S 51$ BImSchG umfassen diese Kreise u.a. Vertreter der Wirtschaft und der Betroffenen. Hier werden anders als nach der GGO zumindest auch die Betroffenen angehört - wenngleich lediglich in repräsentativer Weise, da nur Vertreter der Betroffenen gehört werden. Bei der Auswahl besteht ein weites Ermessen der Bundesregierung oder des federführenden Ministeriums. ${ }^{66}$ Partizipation bezieht sich damit nicht auf alle Betroffenen. Genauere Vorschriften, beispielsweise wie das Vorbringen der Kreise zu berücksichtigen ist, fehlen. Eine gänzlich unterbliebene Anhörung führt anders als bei der GGO zur Nichtigkeit der Rechtsverordnung. ${ }^{67}$ Die Nicht-Anhörung Einzelner führt hingegen nicht zur Nichtigkeit, sofern kein schwerer Verstoß vorliegt. ${ }^{68}$ Es besteht kein unmittelbarer Rechtsschutz im Falle der Nichtbeteiligung. ${ }^{69}$

64 F. Ossenbühl (Fn. 61), Rn. 70.

65 Siehe z.B. $\S 68$ KrWG; $\S 17$ Abs. 7 ChemG; $\S 20$ BBodSchG; $§ 7$ Gesetz über die Umweltverträglichkeit von Wasch- und Reinigungsmitteln.

66 H.-J. Koch, § 51, Rn. 31, in: H.-J. Koch/E. Pache/D. Scheuing (Hrsg.) Gemeinschaftskommentar zum Bundes-Immissionsschutzgesetz, Loseblattsammlung Band III, 35. Lieferung März 2014; C. Tophoven, §51 BImSchG, Rn. 7, in: L. Giesberts/M. Reinhardt (Hrsg.), Beck'scher Online-Kommentar Umweltrecht, Edition 31, Stand 1. Juli 2015.

67 H.-J. Koch (Fn. 66), § 51, Rn. 37; C. Tophoven (Fn. 66), § 51 BImSchG, Rn. 9; H. D. Jarass, Bundes-Immissionsschutzgesetz. Kommentar, 8. Aufl. 2010, §51, Rn. 4; G. Feldhaus, § 51, Anm. 4, in: G. Feldhaus/H.D. Hansel (Hrsg.), Bundesimmissionsschutzrecht. Kommentar, Loseblattsammlung Band 1 - Teil II, 180. Lieferung Februar 2014.

68 H.-J.Koch (Fn. 66), § 51, Rn. 41; J. Tophoven (Fn. 66), § 51 BImSchG, Rn. 9.

69 J. Tophoven (Fn. 66), § 51 BImSchG, Rn. 10. 


\section{(3) Raumordnungsrecht}

In einigen Fällen der Rechtsverordnungsgebung ist sogar eine Öffentlichkeitsbeteiligung - und nicht nur eine Repräsentanten- oder Betroffenenbeteiligung i.S. einer Beteiligung beteiligter Kreise - zwingend vom Gesetzgeber vorgesehen. So ist die Öffentlichkeit bei der Erstellung von Raumordnungsplänen, die auf Bundesebene vom Bundesverkehrsministerium immer und auf Landesebene oftmals als Rechtsverordnung ${ }^{70}$ erlassen werden, nach $\S 10$ Abs. 1 ROG (für den Bund i.V.m. § 18 ROG) zu beteiligen.

Wie findet hier die Beteiligung statt? Zunächst ist die Öffentlichkeit von der Aufstellung des Raumordnungsplans zu unterrichten. Dies kann durch öffentliche Auslegung des Entwurfs oder auch auf andere Art und Weise, etwa innerhalb eines Erörterungstermins geschehen. ${ }^{71}$ Nach der Unterrichtung ist der Öffentlichkeit Gelegenheit zur Stellungnahme zu geben. Nach $\S 7$ Abs. 2 S. 2 ROG sind die Stellungnahmen in den Beteiligungsverfahren im Rahmen der Abwägung zu berücksichtigen. Unmittelbarer Rechtsschutz gegen Raumordnungspläne kommt nach $\S 43$ VwGO, gegen Landespläne auch nach $\S 47 \mathrm{VwGO}$ in Betracht. ${ }^{72}$ Dabei ist jedoch zu berücksichtigen, dass Verletzungen des Beteiligungserfordernisses bei der Erstellung von Raumordnungsplänen dann nicht beachtlich sind, wenn einzelne Personen nicht beteiligt worden sind und die entsprechenden Belange unerheblich waren oder in der Entscheidung berücksichtigt wurden (§ 12 Abs. 1 ROG). Ein beachtlicher Verfahrensverstoß liegt immer dann vor, wenn eine Beteiligung gar nicht stattgefunden hat. ${ }^{73}$

70 Nach den verschiedenen Landesraumordnungsgesetzen ergehen Landesraumordnungspläne z.T. als Parlamentsgesetz, z.T. als Rechtsverordnung, z.T. im Wege der Feststellung durch die Landesregierung oder die Landesplanungsbehörde. S. den Überblick bei W. Krebs, Baurecht, in: F. Schoch (Hrsg.), Besonderes Verwaltungsrecht, 15. Auflage 2013, Rn. 55.

71 W. Spannowsky, § 12 Rn. 13, in: W. Spannowsky/P. Runkel/K. Goppel (Hrsg.), Raumordnungsgesetz. Kommentar, 2010.

72 R. Danielzyk/K. Hanebeck/J. Knieling/F. Reitzig, Öffentlichkeitsbeteiligung bei Programmen und Plänen der Raumordnung, 2003, $152 \mathrm{f}$.

73 Vgl. dazu W. Spannowsky (Fn. 71), § 12, Rn. 40 ff. 


\section{(4) Bewertung}

Blickt man auf die Beteiligungsmöglichkeiten beim Erlass von Rechtsverordnungen, so fällt zum einen die demokratische Komponente von Beteiligungsrechten auf, zum anderen die Zurückhaltung bezüglich der Frage, wie genau die Beteiligungsergebnisse $\mathrm{zu}$ beachten sind.

Das demokratische Verständnis von Beteiligung zeigt sich darin, dass auf der Ebene der Raumordnungsplanung alle partizipieren dürfen und dies unabhängig von einer möglichen Rechtsverletzung. Dies ist anders im Rahmen der GGO und der Gesetze, die die beteiligten Kreise in das Verfahren mit einbeziehen. Aber gerade die Tatsache, dass hier Einzelne, die unter Umständen in ihren Rechten verletzt sein können, aus diesem Umstand kein Recht auf Beteiligung ableiten können, spricht ebenfalls für eine demokratische Deutung der Beteiligung. Hier zeigt sich nämlich, dass nicht ausschließlich die Rechte des Einzelnen und damit individuelle Selbstbestimmung Gegenstand der Beteiligung sind. Vielmehr soll mit dem Beteiligungsverfahren auch kollektive Selbstbestimmung gestärkt werden: Das Verfahren als Ganzes und damit die Interessen aller und nicht die Rechte Einzelner stehen im Mittelpunkt dieser Beteiligungsvorschriften.

Dem entspricht die Rechtsfolgenseite. Die Rechtsfolge der Nichtigkeit bzw. der Rechtswidrigkeit kommt im Rahmen von Gesetzen, die beteiligte Kreise ermächtigen bzw. im Rahmen von Raumordnungsplänen nämlich nicht etwa schon dann in Betracht, wenn die Rechte Einzelner verletzt wären. Dies würde für eine rein rechtsstaatliche Deutung von Beteiligungsrechten sprechen. ${ }^{74}$ Vielmehr wird auf das Verfahren als Ganzes abgestellt. Dies entspricht einem demokratischen Verständnis von Beteiligung wie ein Vergleich mit der Herangehensweise des Bundesverfassungsgerichts in Bezug auf die Verletzung des - subjektiven - Wahlrechts, dem „Kern“75 von Demokratie verdeutlichen soll. Auch hier unterscheidet man zwischen dem Recht des Einzelnen und dem Verfahren als Ganzem, das Demokratie fördern soll. Wenn z.B. ein Wahlbogen nicht mitgezählt wird, ist das subjektive Recht des Betroffenen vollständig verletzt, die Wahl als solche ist aber nicht ungültig. Wenn die Wahl gar nicht stattfindet oder erhebliche

74 So H.-J. Koch (Fn. 66), § 51, Rn. 37.

75 M. Kriele, Das demokratische Prinzip im Grundgesetz, VVDStRL 29 (1971), 46 (82). 
Mängel aufweist, dann ist das ganze Verfahren - und damit die Staatsgewalt - delegitimiert. ${ }^{76}$

Schließlich zeugen die Regelungslücken bezüglich der Art und Weise der Berücksichtigung von Beteiligung von einem auch demokratischen Verständnis der Beteiligungsformen: Durch das Offenhalten des Entscheidungsprozess wird die (deliberative) Rückbindung des Staates an die Gesellschaft gestärkt.

\section{Planfeststellungsbehörden}

Auch vor dem Erlass von Verwaltungsakten kann eine Öffentlichkeitsbeteiligung zwingend vorgeschrieben sein, etwa im Rahmen von Planfeststellungsverfahren nach $\S 73$ Abs. 4 S. 1 VwVfG.

Das Planfeststellungsverfahren ist rechtlich determinierter als die Rechtsverordnungsgebung und betrifft einen kleineren Kreis an Personen als Rechtsverordnungen, die potentiell alle Bürger betreffen. Das Planfeststellungsverfahren ist aber ebenfalls in die Zukunft gerichtet und betrifft immer noch einen größeren Personenkreis als etwa ein an eine Person gerichteter Verwaltungsakt. Im Vergleich zur Rechtsverordnungsgebung orientiert sich das Planfeststellungsverfahren somit weiter Richtung individuelle Selbstbestimmung. Gleichzeitig kommen aber auch Elemente kollektiver Selbstbestimmung zum Tragen.

Als Planfeststellungsbehörde kommen grundsätzlich alle Behörden der unmittelbaren Staatverwaltung in Betracht. Dies sind neben den obersten Behörden und Bundes- bzw. Landesoberbehörden auch Mittelbehörden und untere Behörden. ${ }^{77}$ Vor dem Erlass von Planfeststellungsbeschlüssen ist nach $\S 73$ Abs. 4 S. 1 VwVfG jeder zu beteiligen, der in seinen Belangen beeinträchtigt ist. Dies ist der Fall, wenn ein schutzwürdiges Interesse rechtlicher, wirtschaftlicher oder ideeller Art berührt ist. ${ }^{78}$ Mögliche

76 Vgl. dazu BVerfGE 85, 148 (157ff.); H. H. Klein, Art. 41, Rn. 99 ff., in: T. Maunz/G. Dürig, Grundgesetzkommentar, Loseblattsammlung Band IV, 70. Ergänzungslieferung Dez. 2013.

77 Vgl. z.B. § 38 Abs. 5 Niedersächsisches Straßengesetz für Gemeindestraßen, Nds. GVB1. 1980, 359, oder $§ 16$ Abs. 2 Seilbahngesetz Sachsen-Anhalt, GVB1. LSA 2012, 526.

78 VGH Kassel, NVwZ 5 (1986), 680 (682). Z.T. wird der Begriff des „Belanges“ sogar noch weiter gefasst. So sollen alle ,wirtschaftlichen, ökologischen, sozialen, kulturellen, ideellen oder sonstigen nicht unredlichen und deshalb anerkennswer- 
Rechtsverletzungen sind keine Beteiligungsvoraussetzung. Der Kreis der Beteiligten ist also möglichst groß, wenngleich nicht ,alle“ beteiligt werden. Diese Einschränkung ist nur folgerichtig, Planfeststellungen und das entsprechende Verfahren orientieren sich näher am rechtsstaatlichen Prinzip als Rechtsverordnungen und die entsprechenden Verfahren: Wie $\S 73$ VwVfG und die Regelung, dass Planfeststellungsbeschlüsse als Verwaltungsakt erlassen werden, zeigen, sind die Regeln zum Planfeststellungsbeschluss rechtlich determinierter als die der Rechtsverordnungsgebung. Auch betreffen sie einen kleineren Kreis an Personen als Rechtsverordnungen, die potentiell alle Bürger betreffen. Die höhere Regelungsdichte ist Ausdruck der stärkeren Rechtsgebundenheit der Behörde.

Aber auch das Demokratieelement ist ausgeprägt: Planfeststellungsbeschlüsse regeln die Zukunft. Das demokratische Element zeigt sich auch am Beteiligungsverfahren und am Gestaltungsspielraum der Planfeststellungsbehörden. Das Beteiligungsverfahren ist ähnlich dem der Aufstellung eines Raumordnungsplans ausgestaltet: ${ }^{79}$ Zunächst muss der Plan ausgelegt werden, darauf folgt die Anhörung, die durch schriftliche Einwendungen erfolgt. ${ }^{80}$ Hinzu kommt grundsätzlich ein Erörterungstermin. ${ }^{81}$ In diesem müssen die Probleme substanziell besprochen werden ${ }^{82}$ mit dem Ziel, eine Einigung zu erzielen. Dieses Ziel folgt aus § 74 Abs. 2 S. 1 VwVfG, nach dem die Behörde in einem abschließenden Schritt über die nicht erledigten Einwendungen zu entscheiden hat. Die Einwendungen müssen „selbstverständlich“ ernsthaft in Erwägung gezogen werden und im Planfeststellungsbeschluss Berücksichtigung finden. ${ }^{83}$ Sie sind mithin in die Abwägung einzubeziehen. ${ }^{84}$ Es besteht aber kein Einigungszwang. Eine

ten eigenen Interessen“ darunter fallen, A. Guckelberger, Formen von Öffentlichkeit und Öffentlichkeitsbeteiligung im Umweltverwaltungsrecht, Verwaltungsarchiv 103 (2012), 31 (44).

79 A. Guckelberger (Fn. 78), 53.

$80 § 73$ Abs. 3-5 VwVfG.

$81 \S 73$ Abs. 6 VwVfG sieht ihn zwingend vor, in manchen der besonderen Planfeststellungsverfahren ist er lediglich fakultativ vorgesehen, siehe z.B. $§ 17$ a Nr. 5 FStrG.

82 M. Knauff, Öffentlichkeitsbeteiligung im Verwaltungsverfahren, DÖV 65 (2012),

1 (5); J. Ziekow, Verwaltungsverfahrensgesetz. Kommentar, 2. Aufl. 2010, § 73 Rn. 62.

83 A. Guckelberger (Fn. 78), 54.

84 W. Neumann, § 74 Rn. 162, in: P. Stelkens/H.J. Bonk/ M. Sachs (Hrsg.), Verwaltungsverfahrensgesetz. Kommentar, 8. Auflage 2014. 
nachvollziehbare Zurückweisung und sachlich begründetete Nichtberücksichtigung von Einwendungen ist statthaft. ${ }^{85}$

Ebenso wie bei der Aufstellung von Raumordnungsplänen zeugt der planerische Gestaltungsspielraum von einer starken demokratischen Ausprägung des Planfeststellungsverfahrens. Er erlaubt der Behörde, die im Beteiligungsverfahren vorgebrachten Interessen - und nicht nur die Rechte - der Einwender ebenso im Wege der Abwägung zu berücksichtigen wie die der Allgemeinheit. ${ }^{86}$ Damit besitzt die Behörde einen gewissen Spielraum und gestaltet dadurch Zukunft.

Auch der Erörterungstermin, der sich als „Kernstück und Höhepunkt der Beteiligung " ${ }^{\text {87 }}$ beschreiben lässt, ist aufgrund seines kommunikativen Charakters sowie der Offenheit des Prozesses Zeichen der demokratischen Ausprägung von Partizipation im Planfeststellungsverfahren.

\section{Ordnungsbehörden}

Am anderen Ende des Bogens steht die Ordnungsbehörde vor Ort, die jedenfalls dann ganz gerichtsähnlich tätig wird, wenn ihr kein Ermessensspielraum zukommt. Ordnet das Ordnungsamt etwa die Beseitigung eines morschen Baums an, so sind subjektive Rechte und nicht Belange und Interessen zu beachten, vor allem die Grundrechte des Störers, vielleicht noch die des Nachbarn. Hier kann sich nur derjenige beteiligen, der in seinen Rechten oder rechtlichen Interessen beeinträchtigt ist, §13 VwVfG. Eine Öffentlichkeitsbeteiligung ist konsequenterweise nicht vorgesehen ${ }^{88}$ und hätte hier auch keine Daseinsberechtigung.

\section{Fazit}

Aus dem vorher Gesagten ergibt sich, dass die heute schon bestehenden Partizipationsverfahren grundsätzlich versuchen, den Bogen zwischen Rechtsstaatlichkeit und Demokratie zu schlagen. Dies ist folgerichtig, denn aus dem Gewaltenteilungsgrundsatz folgt die staatliche Pflicht, eine

85 M. Knauff (Fn. 82), 5.

$86 \S 74$ Abs. 2 VwVfG.

87 I. Appel (Fn. 11), 1363.

88 M. Knauff (Fn. 82), 4. 
entsprechende Balance zu schaffen. Um diesen Ausgleich zu erreichen, müssen die Partizipationsakteure und die ihnen zukommende Entscheidungsmacht prinzipiell entsprechend dem hier vorgeschlagenen Modell konzipiert werden:

Auf Ebene der Judikative dürfen sich nur Einzelne beteiligen, die in ihren Rechten betroffen sind. Die Entscheidungsmacht verbleibt beim Gericht.

Auf Legislativebene muss das Volk neben Personal- auch Sachfragen entscheiden können. Mitwirkungsverfahren unterhalb der Entscheidungsebene stärken Legitimation, wenn alle sich beteiligen dürfen. Vorbilder gibt es in Südafrika und Baden-Württemberg.

Auf Ebene der Exekutive bedarf es einer genauen Analyse, ob Rechtsstaatlichkeit oder demokratische Willensbildung im Vordergrund stehen. Die Entscheidung wird unabhängig davon immer vom staatlichen Organ getroffen. Die Interessen der Bürger können stärker berücksichtigt werden, wenn der demokratische Charakter des Verfahrens im Vordergrund steht und müssen weniger bis gar nicht berücksichtigt werden, wenn der rechtsstaatliche Charakter des Verfahrens im Vordergrund steht. Die Rechte der Bürger müssen selbstverständlich immer beachtet werden. Parallel verläuft die Bestimmung der zu Beteiligenden. Sie verläuft von ,allen“ über diejenigen, die in ihren Belangen berührt sind, hin zu denjenigen, die in ihren Rechten betroffen sind.

\section{E. Ausblick}

Im Wege eines Ausblicks soll ein schlaglichtartiger Blick auf die Konsequenzen des hier vorgelegten Konzepts geworfen werden. Im Laufe des Aufsatzes war immer wieder von Defiziten die Rede. Die Defizite beziehen sich einerseits auf die Rechtsanwendung, andererseits auf die Gesetzgebung.

Die Crux dieses Ausblicks soll nicht verschwiegen, sondern ihm vielmehr vorangestellt werden: Sind Rechtsstaats- und vor allem Demokratieprinzip Regeln, Prinzipien, Optimierungsgebote, Staatsstrukturbestimmungen, Staatsziele und/oder Staatsaufgaben? ${ }^{89}$ Das ist umstritten und

89 Siehe die vielen weiteren Bezeichnungen bei K. Nowrot, Das Republikprinzip in der Rechtsordnungengemeinschaft. Methodische Annäherungen an die Normalität eines Verfassungsprinzips, 2014, 69 ff. mwN. 
noch mehr: was aus einer solchen Klassifikation genau folgt, ist ebenfalls unklar. ${ }^{90}$ Aufgrund der offenen und nicht lediglich binären Strukturierung von Rechtsstaats- und Demokratieprinzip wird hier davon ausgegangen, dass sie Staatszielbestimmungen sind. ${ }^{91}$ Rechtsstaats- und Demokratieprinzip sehen nämlich kein entweder/oder vor und ordnen keine Rechtsfolge für ein bestimmtes Verhalten an, ja, sie kennen nicht einmal den Unterschied zwischen Tatbestand und Rechtsfolge. Zwar kann ein staatliches Verhalten gegen das Demokratie- oder das Rechtsstaatsprinzip verstoßen, es also nicht erfüllen, ein anderes Verhalten hingegen erfasst sein. ${ }^{92}$ Anders aber als bei ausschließlich binär strukturierten Normen sind hinsichtlich des Demokratieprinzips wie des Rechtsstaatsprinzips sowohl aufgrund ihres Inhalts als auch aufgrund ihrer Normstruktur unterschiedliche Verwirklichungsstufen denkbar. ${ }^{93}$ Schon aufgrund der Existenz des Art. 79 Abs. 3 GG, der den Kernbereich des Demokratie- und Rechtsstaatsprinzips auch für den Fall einer Verfassungsänderung für unantastbar erklärt und damit zwischen veränderungsfesten und veränderbaren Gehalten von Demokratie- und Rechtsstaatsprinzip differenziert, wird man annehmen müssen, dass das Grundgesetz in Art. 20 GG nicht nur ein Mindestmaß an

90 Siehe ausführlich K.-P. Sommermann, Art. 20, Rn. 5, 88 ff., in: H. von Mangoldt/F. Klein/C. Starck (Hrsg.), Grundgesetz-Kommentar, Band 2, 6. Auflage 2010 und F. Reimer, Verfassungsprinzipien. Ein Normtyp im Grundgesetz, 2001, 183 ff. und passim; U. Volkmann, Art. 20 (Demokratieprinzip), Rn. 37 ff., in: K.-H. Friauf/W. Höfling (Hrsg.), Berliner Kommentar zum Grundgesetz, Loseblatt, Stand 2015.

91 Ausdrücklich für Rechtsstaatsprinzip: U. Scheuner, Staatszielbestimmungen, in: R. Schnur (Hrsg.), Festschrift für Ernst Forsthoff, 1972, 325 (335 f.); K. Nowrot (Fn. 89), 384; P. Kunig, Das Rechtsstaatsprinzip, 1986, 185; ausdrücklich für Demokratieprinzip: A. von Komorowski, Demokratieprinzip und Europäische Union. Staatsverfassungsrechtliche Anforderungen an die demokratische Legitimation der der EG-Normsetzung, 2010, 587; U. Volkmann (Fn. 90), Rn. 38, der das Demokratieprinzip darüberhinaus als Programm bezeichnet; $W$. Thieme, Demokratie - Ein Staatsziel im Wandel der gelebten Verfassung, DÖV 1998, 751; A. Bleckmann, Vom Sinn und Zweck des Demokratieprinzips, 1998, 124; a.A. K.-P. Sommermann (Fn. 90), Rn. 91.

92 Vgl. R. Poscher, Theorie eines Phantoms. Die erfolglose Suche der Prinzipientheorie nach ihrem Gegenstand, Rechtswissenschaft, 2010, $349 \mathrm{ff}$. Siehe auch die Nachweise bei K. Nowrot (Fn. 89), 511 f.

93 U. Schliesky, Souveränität und Legitimation von Herrschaftsgewalt, 2004, 617 für das Demokratieprinzip; hinsichtlich des Rechtsstaatprinzips zeigt dies schon die Unterscheidung zwischem materiellen und formellen Rechtsstaatsbegriff, siehe dazu E. Schmidt-Aßmann, Der Rechtsstaat (§ 23), in: J. Isensee/P. Kirchhof (Hrsg.), Handbuch des Staatsrechts, Band I, 3. Aufl. 2004, Rn. 18. 
Demokratie und Rechtsstaatlichkeit garantiert - diese Garantie findet sich nämlich schon in Art. 79 Abs. 3 GG. Demokratie- und Rechtsstaatsprinzip sind damit dynamisch und beziehen sich auf materiell-inhaltliche Ziele. Daher muss sich das Grundgesetz gezwungenermaßen zu der Frage verhalten, welche Verwirklichungsstufe erreicht werden soll. Dies tut es durch die Regelungstechnik der Staatszielbestimmung, die alle drei Gewalten verpflichtet, auf die Zielerreichung hinzuwirken. Die Zielerreichung muss unter Berücksichtigung anderer Normen und Verfassungsprinzipien erfolgen. Entsprechend dem Gebot der Herstellung praktischer Konkordanz $^{94}$ im Bereich der Grundrechtsauslegung, erscheint es auch hier überzeugend, im Rahmen des Ausgleichs verschiedener Verfassungsziele ein Optimum - das von einem Maximum zu unterscheiden ist ${ }^{95}$ - an Zielverwirklichung zu verlangen. Dementsprechend lässt sich von einem Optimierungsgebot sprechen. ${ }^{96}$

\section{Rechtsanwendung}

Schaut man auf die Rechtsanwendung, so verpflichtet das hier vertretene Verständnis von Gewaltenteilung, Demokratie und Rechtsstaat die Verwal-

94 Siehe zu diesem Zusammenhang schon F. Müller, Normstruktur und Normativität. Zum Verhältnis von Recht und Wirklichkeit in der juristischen Hermeneutik, entwickelt an Fragen der Verfassungsinterpretation, 1966, 213.

95 M. Morlok, Demokratie und Wahlen, in: Peter Badura/Horst Dreier (Hrsg.), Festschrift 50 Jahre Bundesverfassungsgericht, Bd. 2, 2001, 559 (564).

96 Explizit das Demokratieprinzip als Optimierungsgebot bezeichnend U. Schliesky (Fn. 93), 616 ff.; B.-O. Bryde, Das Demokratieprinzip des Grundgesetzes als Optimierungsaufgabe, in: Redaktion Kritische Justiz (Hrsg.), Demokratie und Grundgesetz, 2000, 59 ff.; M. Morlok (Fn. 95), 562 f.; A. von Bogdandy (Fn. 11), 30; H.H. Trute, Die demokratische Legitimation der Verwaltung (§ 16), in: W. Hoffmann-Riem/E. Schmidt-Aßmann/A. Voßkuhle (Hrsg.), Grundlagen des Verwaltungsrechts Band I, 2. Aufl. 2012, Rn. 16; E. Pache, Abschließender Beitrag in der Aussprache zu seinem Vortrag Verantwortung und Effizienz in der Mehrebenenverwaltung, VVDStRL 66 (2007), 209 (213); C. Calliess, Bürgerrechte als Ersatz für Demokratie?, in: C. Franzius/F. C. Mayer/J. Neyer (Hrsg.), Strukturfragen der Europäischen Union, 2010, 231 (239). 
tung, ihren Auslegungsspielraum ${ }^{97}$ und ihr Ermessen ${ }^{98}$ dahingehend auszuüben, Partizipation zu ermöglichen. So hat die Verwaltung beispielsweise in den Fällen, in denen der Erörterungstermin lediglich fakultativ vorgesehen ist, ihr Ermessen grundsätzlich dahingehend ausüben, dass dieser stattzufinden hat. ${ }^{99}$

Die Judikative ist aufgefordert, Beteiligungsrechte zu schützen. So sollte sie von der bisherigen Auslegung des $\S 46$ VwVfG abrücken. ${ }^{100} \S 46$ VwVfG sieht vor, dass eine Verletzung von Beteiligungsrechten im Verwaltungsverfahren unerheblich ist, sofern offensichtlich ist, dass die Verletzung die Entscheidung in der Sache nicht beeinflusst hat. Die Gerichte gehen zumeist davon aus, dass im konkreten Fall die Beteiligung zu keinem anderen Ergebnis geführt hätte. Jedoch besteht aufgrund des demokratischen Charakters von Beteiligung abstrakt immer die Möglichkeit, dass Kommunikation und Diskurs zwischen Behörde und Bürgern zu einem anderen Ergebnis führen können. ${ }^{101}$ Dementsprechend sollte $\S 46$ VwVfG ausgelegt werden und damit bei der Verletzung von Beteiligungsrechten nicht einschlägig sein. ${ }^{102}$

97 Bundesministerium des Inneren/Bundesministerium der Justiz (Hrsg.), Bericht der Sachverständigenkommission Staatszielbestimmungen/Gesetzgebungsaufträge, 1983, 21; A. von Komorowski (Fn. 91), 588; W. Abendroth, Zum Begriff des demokratischen und sozialen Rechtsstaats im Grundgesetz der Bundesrepublik Deutschland, in: A. Hermann (Hrsg.), Aus Geschichte und Politik. Festschrift zum 70. Geburtstag von Ludwig Bergstraesser, 1954, 279 (281); I. Contiades, Verfassungsgesetzliche Staatsstrukturbestimmungen, 1967, 117; E. Wienholtz, Arbeit, Kultur und Umwelt als Gegenstände verfassungsrechtlicher Staatszielbestimmungen, Archiv des öffentlichen Rechts 109 (1984), 532 (536).

98 So allgemein für Staatsstrukturbestimmungen BVerwG, Urteil vom 9. November 1978 - 3 C 68.77 = Buchholz 427.3 § 335a LAG Nr. 63 Rn. 27 (juris); I. Contiades (Fn. 97), 117.

99 Vgl. auch die Kritik bei M. Wickel, §39 Fachplanung, in: D. Ehlers/M. Fehling/H. Pünder (Hrsg.), Besonderes Verwaltungsrecht, Bd. 2, 3. Aufl. 2013, Rn. $39 \mathrm{ff}$.

100 Ebenso V. M. Haug/K. Schadtle, Der Eigenwert der Öffentlichkeitsbeteiligung im Planungsrecht. Zugleich ein Beitrag zur Dogmatik des § 46 VwVfG, NVwZ 33 (2014), 271 (275). Zu den Verfassungsprinzipien als Auslegungsregel siehe BVerfGE 9, 231 (262); I. Contiades (Fn. 97), 117 (spricht von Staatsstrukturbestimmung); W. Abendroth (Fn. 97), 281.

101 Siehe die ausführliche Kritik bei A. Fisahn (Fn. 10), 349 ff. mzN.

102 Vgl. auch M. Gellermann, Verbandsklagen im Umweltrecht - aktueller Stand, Perspektiven und praktische Probleme, DVB1. 2013, 1341 (1345); BVerwG, 
Hier soll keinesfalls einer umfassenden verwaltungsgerichtlichen Kontrolle über den Hebel der Beteiligungsrechte das Wort geredet werden. So wird zwar durch Beteiligungsrechte die Klagebefugnis erweitert, da diese als subjektive Rechte verstanden werden müssen. ${ }^{103}$ Dies darf aber nicht dazu führen, dass dadurch der materielle Prüfungsumfang des Gerichts auch auf darüber hinaus liegende Fragen, die nicht die Verletzung subjektiver (Beteiligungs-)Rechte des Klägers betreffen, erweitert wird. Dies wäre ein Übergriff der Judikative in die demokratische Willensbildung der Verwaltung. Dass dies nicht die Folge des Vorschlags ist, folgt schon aus $\S 113$ Abs. 1 S. 1 VwGO - und aus dem Grundsatz der Gewaltenteilung: Gerichte dürfen lediglich den Kläger vor einer subjektiven Rechtsverletzung schützen und nicht eine allgemeine Rechtmäßigkeitskontrolle vornehmen.

\section{Gesetzgebung}

Die Gesetzgebung darf bei der Erweiterung und Neuschaffung von Beteiligungsformen nicht gegen Demokratieprinzip, Rechtsstaatsprinzip und

NVwZ 2012, 557 (558), Rn. 18; EuGH, Urteil vom 7. November 2013, C-72/12 - Altrip, Rn. 48.

103 Ebenso K. F. Gärditz, Verwaltungsgerichtlicher Rechtsschutz im Umweltrecht, NVwZ 2014, 1 (3); M. Ogorek, Die Anfechtung von Planfeststellungsbeschlüssen durch Gemeinden nach Inkrafttreten des Umwelt-Rechtsbehelfsgesetzes, NVwZ 2010, 401 (402 f.); W. Kahl, Neuere höchstrichterliche Rechtsprechung zum Umweltrecht - Teil 1, JZ 2014, 722 (732); J. Ziekow, Verfahrensfehler im Umweltrecht - notwendige Nachjustierungen im deutschen Verwaltungsrecht, NuR 2014, 229, 234; OVG Koblenz, NVwZ 2005, 1208 (1210f.); D. Murswiek/L. Ketterer/O. Sauer/H. Wöckel, Rechtsprechungsanalyse. Ausgewählte Probleme des Umweltrechts - Subjektivierungstendenzen; Umweltinformationsrecht, Die Verwaltung 44 (2011), 235 (250); M. Kment, Das neue Umwelt-Rechtsbehelfsgesetz und seine Bedeutung für das UVPG - Rechtsschutz des Vorhabenträgers, anerkannter Vereinigungen und Dritter, NVwZ 2007, 274 (279); offenlassend, aber aufgeschlossen OVG Münster, Beschluss vom 23. Juli 2014, 8 B 356/14, BeckRS 2014, 54136, 2 a); ablehnend K.-P. Dolde, Verwaltungsverfahren und Deregulierung, NVwZ 2006, 857 (861); H. Lecheler, Isolierte Anfechtung von Verfahrensfehlern ohne materielle Beschwer kraft Europarechts?, NVwZ 2005, 1156 f. Siehe ausführlich D. Steiger, Entgrenzte Gerichte? Die Ausweitung des subjektiven Rechts und der richterlichen Kontrollbefugnisse - Verwaltung und Parlament im „Kooperationsverhältnis“ der deutschen Verwaltungs- und Verfassungsgerichtsbarkeit mit dem EuGH, erscheint Verwaltungsarchiv 2016. 
Gewaltenteilungsprinzip verstoßen. So dürfte die Legislative beispielsweise nicht einen Betroffenenentscheid auf Verwaltungsebene einführen, also Betroffene statt der Verwaltung in quasi-legislativer Manier entscheiden lassen. ${ }^{104}$ Dies würde nicht nur nach dem „Legitimationskettenmodell“105 dem Demokratieprinzip widersprechen, sondern auch gegen das Gewaltenteilungsprinzip verstoßen, weil so Aufgaben der Verwaltung durch eine quasi-legislative Entscheidung wahrgenommen werden würden. Außerdem kann es zu einem Konflikt mit dem Rechtsstaatsprinzip kommen, wenn Individualrechte Dritter betroffen sind.

Versteht man wie hier Demokratie- und Rechtsstaatsprinzip als Staatszielbestimmung und Optimierungsgebot, so wird das Parlament in die Pflicht genommen, Partizipationsmöglichkeiten zu erweitern und entsprechend dem hier entwickelten Konzept zu gestalten. Dies betrifft u.a. die Einführung von Elementen direkter Demokratie auf Bundesebene. ${ }^{106}$ Bei deren Einführung durch den verfassungsändernden Gesetzgeber muss beachtet werden, dass auch das Volk an Grundrechte und Rechtsstaatlichkeit gebunden ist. ${ }^{107}$ Das Parlament ist ebenso gehalten, erweiterte Einflussnahmemöglichkeiten des Einzelnen auf die formelle und die materielle Gesetzgebung zu schaffen. Hier sind Beteiligungsmöglichkeiten zu eröffnen, die allen Bürgern der jeweiligen Verbandsebene offenstehen und nicht nur Repräsentanten der Wirtschaft. Nur dies entspricht dem vor-

104 D. Steiger, Mehr Direkte Demokratie? Gewagt!, in: Juristische Ausbildung 2014, 963 (973 ff.); W. Ewer, Kein Volksentscheid über die Zulassung von Infrastrukturprojekten, NJW 2011, 1328 (1329); W. Durner, Möglichkeiten der Verbesserung förmlicher Verwaltungsverfahren am Beispiel der Planfeststellung, ZUR 2011, 354 (361); R. Steinberg, Die Bewältigung von Infrastrukurvorhaben durch Verwaltungsverfahren - eine Bilanz, ZUR 2011, 340 (348 f.).

105 BVerfGE 83, 37 (51); 83, 60 (71 ff.); 93, 37 (68 ff.); siehe aber auch BVerfGE 107, 59 (87 ff.); E.-W. Böckenförde, Demokratie als Verfassungsprinzip (§ 24) in: J. Isensee/P. Kirchhof (Hrsg.), Handbuch des Staatsrechts Band II, 3. Aufl. 2004, Rn. 11 ff. Siehe auch die Kritik daran bei B.-O Bryde, Die bundesrepublikanische Volksdemokratie als Irrweg der Demokratietheorie, StWStP 5 (1994), 305; T. Groß, Das Kollegialprinzip in der Verwaltungsorganisation, 1999, 166 ff.; G. Haverkate, Verfassungslehre, 1992, $330 \mathrm{ff}$.

106 Ebenso u.a. C. Pestalozza, Der Popularvorbehalt, Direkte Demokratie in Deutschland, 1981, $12 \mathrm{ff}$.

107 Vgl. zu dieser Frage B. Hartmann, Volksgesetzgebung und Grundrechte, 2005; G. Kirchgässner, Direkte Demokratie und Menschenrechte, in: L. Feld/P.M. Huber/O. Jung/C. Welzel/F. Wittreck (Hrsg.), Jahrbuch Direkte Demokratie 2009, 66. 
nehmlich demokratischen Charakter des materiellen Gesetzeserlasses, aber auch des Rechtsverordnungserlasses durch die Regierung. Für die Ebene des Parlamentsgesetzes können die entsprechenden Regelungen in Südafrika und Baden-Württemberg als Vorbilder dienen. ${ }^{108}$

Dass der Gesetzgeber die Nicht-Öffentlichkeit des Erörterungstermins vorschreibt ${ }^{109}$ und ihn in vielen Fachgesetzen gar fakultativ stellt oder gar nicht vorsieht, entspricht nicht seinem demokratischen Gehalt. Hier besteht gesetzlicher Handlungsbedarf, der Erörterungstermin sollte zwingend und öffentlich sein, so wie es schon $\S 18$ Abs. 1 S. 1 9. BImSchV vorsieht.

Auch lässt sich daran denken, eine Norm wie die des $\S 85$ WHG auch auf andere Rechtsgebiete zu übertragen. Dieser verlangt für die Aufstellung, Überprüfung und Aktualisierung der Maßnahmenprogramme und Bewirtschaftungspläne die Förderung von aktiver Beteiligung durch die zuständige Behörde. Aus einem demokratischen Verständnis von Partizipation folgt auch, dass Bürgerbeteiligung möglichst früh ansetzen muss. Auch muss die Auslegungsfrist von Planentwürfen lang genug sein, damit die Bürger den Diskurs auf Augenhöhe mit dem Staat führen können.

Wird Partizipation entsprechend dem hier vorgestellten Konzept ausgestaltet, so ist die von der Gewaltengliederung geforderte Balance zwischen Demokratie und Rechtsstaatlichkeit erreicht. Mehr Partizipationsmöglichkeiten in diesem Sinne stärken Demokratie und Rechtsstaatlichkeit sowie die Effektivität staatlichen Handelns.

108 Siehe die Beiträge von B. Loots (Fn. 56) und F. Reidinger (Fn. 57) in diesem Band.

$109 \S 73$ Abs. 6 S. 6 i.V.m. $§ 68$ Abs. 1 S. 1 VwVfG. 
